\title{
Actualización sobre factores de riesgo para cefalea pospunción dural
}

\author{
lader Alfonso Rodríguez-Márquez \\ Nayibe Saab-Ortega* \\ * Médico. Especialista en epidemiologia clínica. Universidad Autónoma de Bucaramanga. Bucaramanga. Santander. Colombia.
}

Correspondencia: Dr. lader A. Rodríguez M. Dirección: Circunvalar 36a N 104-25 Torre 2 Apto 504 Bucaramanga. Santander. Colombia. Correo electrónico: irodriguez73@unab.edu.co.

\section{RESUMEN}

Introducción: La cefalea pospunción dural es la complicación más frecuente de la punción dural, reportando una incidencia que oscila entre el 10\% y el 40\%. El padre de la anestesia epidural, August Bier, reportó en 1899 el primer caso de cefalea pospunción dural atribuyéndola a la perdida de líquido cefalorraquideo. Existen dos teorías para explicar su origen; la primera es la teoría de la grieta y la segunda es la teoría de Monro-Kellie-Burrows. El conocimiento de los factores de riesgo permite predecir la probabilidad de desarrollar esta patología. Objetivo: Revisar la evidencia actual sobre los factores de riesgo implicados en su desarrollo. Metodología de búsqueda: Se realizó una búsqueda de la literatura en PubMed, Proquest, Biblioteca Virtual en Salud en el periodo comprendido entre el 14 y 30 de Noviembre del 2014. Conclusiones: La edad, sexo femenino, antecedente personal de cefalea pospunción dural, aguja traumática y orientación del bisel perpendicular son factores de riesgo con significancia estadística. Mientras que los resultados a partir de los estudios no son concluyentes para factores como bajo peso $\left(\mathrm{IMC}<25 \mathrm{Kg} / \mathrm{m}^{2}\right)$, antecedente personal de cefalea, mayor calibre de la aguja, no reinserción del estilete y mayor número de intentos por punción. MÉD UIS. 2015;28(3):345-52.

Palabras Clave: Cefalea Pospunción de la Duramadre. Factores de Riesgo. Causalidad.

\section{Update on risk factors for post-dural puncture headache}

\section{ABSTRACT}

Introduction: Post-dural puncture headache is the most common complication of dural puncture, reporting an incidence ranging between $10 \%$ and $40 \%$. The father of epidural anesthesia, August Bier, reported the first case of post-dural puncture headache in 1899 , attributing it to cerebrospinal fluid loss. There are two theories to explain its origin; the first is the theory of the crack and the second is the theory of Monro-Kellie-Burrows. Knowledge of risk factors allows to predict the likelihood of developing this disease. Objective: To review the current evidence pertaining to the risk factors involved in its development. Research methodology: Conducted literature research in PubMed, ProQuest, and Virtual Library of Health in the period between the 14th and 30th of November of 2014. Conclusions: Age, female gender, personal history of post-dural puncture headache, traumatic needle and orientation of perpendicular bevel are all risk factors with statistical significance. While results from other studies were inconclusive for factors such as low weight $\left(\mathrm{BMl}<25 \mathrm{Kg} / \mathrm{m}^{2}\right)$, personal history of headaches, increased size of needle, no reinsertion of stylet and a high number of attempts to puncture. MÉD UIS. 2015;28(3):345-52.

Keywords: Post-Dural Puncture Headache. Risk Factors. Causality.

¿Cómo citar este artículo?: Rodríguez-Márquez IA, Saab-Ortega N. Actualización sobre factores de riesgo para cefalea pospunción dural. MÉD UIS. 2015;28(3):345-52. 


\section{INTRODUCCIÓN}

La cefalea es la complicación más frecuente de la punción dural, llamada también punción lumbar o punción espinal, independientemente de cual sea su propósito, ya sea para diagnosticar, practicar un tratamiento o simplemente realizar una anestesia espinal ${ }^{1-4}$. Los estudios reportan una gran variabilidad en la incidencia de la Cefalea Pospunción Dural (CPPD) ya que depende de factores de riesgos asociados 5 así como de la clasificación de criterios diagnósticos que se utilice para la CPPD. En términos generales la literatura reporta una incidencia entre el $10 \%$ al $40 \%{ }^{6}$. Kuntz KM y cols ${ }^{7}$, en su estudio reportaron una incidencia de CPPD del 36,5\%; sin embargo, Almeida SM y cols $^{8}$ reportaron una incidencia de $0,9 \%$. Esta baja incidencia podría estar explicada por el uso de agujas atraumaticas calibre 22 y la experiencia de los operadores durante el estudio.

El padre de la anestesia epidural, August Bier, reportó en 1899 el primer caso de CPPD atribuyéndola a la perdida de Líquido Cefalorraquideo (LCR) y la primera publicación de esta teoría la hizo MacRobert en 1918. A principios del siglo XX, la punción dural se realizaba con agujas gruesas, siendo la incidencia de CPPD entre el 50\% y el 60\%. En 1951, Whitacre y Hart desarrollaron la aguja con extremo "en punta de lápiz", con una reducción significativa de la frecuencia de cefalea?.

En la actualidad existen dos teorías para explicar su origen; la primera es la teoría de la grieta, en donde la rasgadura de la duramadre provoca la salida del LCR del espacio subaracnoideo, lo cual lleva a una pérdida de presión y volumen, y por gravedad realiza tracción de estructuras sensibles al dolor como son los senos durales y las venas tributarias, produciendo la cefalea ${ }^{2,3,10}$. La segunda es la teoría de Monro-Kellie-Burrows, la cual consiste en que la suma de los volúmenes del LCR, la sangre y el tejido cerebral se mantienen constantes. Por lo tanto, la pérdida del LCR ocasiona una vasodilatación intracraneal compensatoria mediada por el receptor de adenosina, debido a la estimulación de los nervios sensoriales peri-vasculares que rodean las venas intracraneales y los senos durales; apoyándose así esta hipótesis de vasodilatación venosa intracraneal como la causa de la cefalea ${ }^{3,10,11}$.

La Sociedad Internacional de Cefalea es una organización conformada por profesionales que trabajan en la generación de conocimiento sobre desordenes de dolor de cabeza, la cual publicó en julio del 2013 la tercera edición (versión beta) de la clasificación internacional de desórdenes de cefalea, donde se establece de forma simplificada los criterios diagnósticos para CPPD'2, a diferencia de la versión $a$ anterior $^{13}$ (Ver Tabla 1). Además de los criterios diagnósticos, la CPPD presenta unas características clínicas y factores de riesgo que apoyan su sustento diagnóstico ${ }^{1,14}$. Suele aparecer o empeorar cuando el paciente pasa de una posición supina a una vertical y puede asociarse a lumbago, vértigo, tinnitus, cambios en la audición, parálisis de los nervios craneales, diplopía, ceguera, náuseas, fotofobia (Ver Tabla 2).

La CPPD es una complicación iatrogénica que genera bastante incapacidad, obliga a la consulta por el servicio de urgencias, puede ser origen de complicaciones médicas potencialmente graves y tener incluso implicaciones legales. Conocer los factores de riesgo es importante, ya que permitiría identificar pacientes con alta probabilidad de desarrollar la enfermedad y por ende se podrian utilizar tratamientos profilácticos. El presente artículo pretende revisar la evidencia actual sobre los factores de riesgo implicados en el desarrollo de la CPPD.

Tabla 1. Criterios Diagnósticos ICHD-3 beta ${ }^{a}$

\begin{tabular}{|c|l|}
\hline A & Cualquier cefalea que cumpla el criterio C \\
\hline B & Se halla realizado una punción dural \\
\hline C & $\begin{array}{l}\text { Cefalea desarrollada dentro de los } \\
\text { primeros 5 días de la punción dural }\end{array}$ \\
\hline D & $\begin{array}{l}\text { El cuadro clínico no se explique mejor por } \\
\text { otro criterio diagnóstico del ICHD-3 beta }\end{array}$ \\
\hline
\end{tabular}

Fuente: aThe International Classification of Headache Disorders, 3rd edition (beta version).

\section{Metodología de Búsqueda}

Se realizó una búsqueda de literatura médica para conocer el estado del arte de la CPPD y sus factores de riesgo en bases de datos como PubMed, Proquest y Biblioteca Virtual en Salud. La búsqueda se inció el 14 de Noviembre del 2014 y se incluyeron artículos hasta el 30 de Noviembre del 2014. Se utilizaron estrategias de búsqueda con los siguientes términos del vocabulario MeSH: Post-Dural Puncture Headache, Risk Factors y Causality; vocabulario DeCS: Cefalea Pospunción de la Duramadre, Factores de Riesgo y Causalidad. Se aplicaron los siguientes criterios de inclusión: artículos en idioma inglés y español, publicados en los últimos 10 años y realizados en 
humanos. El criterio de exclusión fue artículos cuyo contenido era sobre el tratamiento de la CPPD. Para lectura crítica en texto completo se escogieron 22 artículos y se adicionaron 28 artículos más procedentes de las citaciones de los documentos iniciales, los cuales se consideraron relevantes para la revisión de tema.

Tabla 2. Características clínicas de la CPPDa

\begin{tabular}{|l|l|}
\hline Aparición & $\begin{array}{l}90 \% \text { de los casos dentro de las 72 } \\
\text { horas después de la punción dural }\end{array}$ \\
\hline Localización & $\begin{array}{l}\text { Bilateral, frontal u occipital, aunque } \\
\text { puede ser generalizada }\end{array}$ \\
\hline Carácter & Opresiva o pulsátil profunda \\
\hline Irradiación & Cuello y hombro \\
\hline Agravante & $\begin{array}{l}\text { Movimientos de la cabeza y } \\
\text { maniobras que aumentan la presión } \\
\text { intracraneal como toser y estornudar. }\end{array}$ \\
\hline Atenuante & Posición horizontal \\
\hline Periodicidad & Asociada a ortostatismo \\
\hline Concomitante & Fotofobia y tinnitus \\
\hline
\end{tabular}

a Cefalea post punción dural

Fuente: Autores.

\section{Factores de Riesgo}

\section{EDAD}

Las personas entre los 20 y 40 años tienen un mayor riesgo de CPPD, presentandose con una incidencia entre tres y cinco veces mayor comparado con personas mayores de 40 años, siendo esta patología escasa en mayores de 60 años. Existen tres posibles factores que podrían evitar el desarrollo de CPPD en mayores de 40 años, la primera, la reducción de elasticidad de la duramadre, lo cual hace que sea más difícil que el LCR se filtre a través del orificio de punción; la segunda, una débil reacción de los vasos cerebrales a la hipotensión del LCR y finalmente, un espacio extradural vertebral reducido que detiene la fuga del LCR del espacio subaracnoideo, aumentando la resistencia extradural ${ }^{2,3,15,16}$. En un estudio realizado por Vandam LD y cols, con 9277 pacientes quienes recibieron anestesia espinal, la incidencia de CPPD en el grupo de edad comprendido entre 20 a 29 años fue del $16 \%$, en el grupo comprendido entre los 40 a 49 años fue del $8 \%$ y en el grupo entre los 50 a 59 años fue del $4 \%$. Zetterberg $\mathrm{H}$ y cols ${ }^{17}$, realizaron un estudio en 1089 pacientes con demencia a quienes se les realizó punciones durales con fines diagnósticos encontrando una baja incidencia de CPPD (2,6\%); sin embargo, la media de la edad de la población de estudio fue de $72 \pm 9,7$ años. Con base en lo anterior, se puede decir que a medida que aumenta la edad, la incidencia de CPPD disminuye ${ }^{3}$.

La CPPD es poco frecuente en los niños ${ }^{18}$, no obstante los estudios muestran que la incidencia oscila entre el $2 \%$ al $15 \%$ en este grupo etario ${ }^{15}$. Hay muy pocos casos documentados ya que la mayoría de los estudios se han realizado en población adulta y los estudios llevados a cabo en este grupo han sido en su mayoría, en población oncológica. Ramamoorthy C y cols ${ }^{19}$, realizaron un estudio en 80 pacientes oncológicos pediátricos en el cual encontraron una incidencia del $8 \%$, con $3 \%$ en menores de 6 años y $5 \%$ entre los 6 a 12 años. Estos resultados se podrían explicar por dos hipótesis, la primera sostiene que la presión hidrostática del LCR en la región lumbar en la población pediátrica es menor comparado con la población adulta y la segunda hipótesis, que en esta población es más difícil el reporte de la información clínica, conllevando a sesgo de información ${ }^{3,15}$ (Ver Tabla 3).

\section{SEXO}

Las mujeres tienen una tendencia mayor de presentar CPPD en comparación con los hombres en una razón de 2:1. Wu CL y $\operatorname{cols}^{20}$, realizaron una revisión sistemática en el 2006 encontrando una incidencia menor en hombres con respecto a las mujeres (OR: 0,55; IC 95\%: 0,44-0,67; p<0,00001). Un estudio prospectivo realizado en Londres por Vilming ST y cols, encontraron una mayor prevalencia en mujeres en una proporción del $46 \%$ versus $21 \%$ $(p=0,0003)^{21}$. Estos datos son explicados por los altos niveles de estrógenos que pueden influenciar el tono de los vasos cerebrales ocasionando distensión de los mismos. Las mujeres procesan la información nociceptiva diferente en comparación con los hombres, mostrando una mayor sensibilidad a estímulos dolorosos ${ }^{2,3,15}$ (Ver Tabla 3).

\section{ÍNDICE DE MASA CORPORAL}

En un estudio realizado por Kuntz KM y cols con 501 pacientes, se encontró un mayor riesgo de desarrollar CPPD en mujeres jóvenes con bajo peso ${ }^{6}$, además en otro estudio, Almeida SM y cols con una muestra de 675 pacientes, entre ellos 575 con diagnóstico de $\mathrm{VIH}$ positivo y 100 controles VIH negativos, se concluyó que el bajo peso es un factor de riesgo para CPPD (OR: 3,26; IC 95\%: 1,53-6,96; 
$p=0,001)^{7}$. El punto de corte que consideraron estos estudios como bajo peso fue IMC menor de $25 \mathrm{Kg} /$ $\mathrm{m}^{2}$, lo cual podría ser un sesgo de estos datos junto con el grupo poblacional que eligieron (pacientes VIH positivos) $)^{2,3,6,7,15}$. Por otro lado, Vricella LK y cols ${ }^{22}$ y Singh S y cols ${ }^{11}$ encontraron en sus estudios mayor riesgo de complicaciones anestésicas durante la cesárea en mujeres gestantes obesas, mientras que otros autores ${ }^{23}$ reportan una incidencia alta de CPPD en la obesas no mórbidas comparada con las obesas mórbidas (45\% versus $25 \%$ ) gestantes con punciones durales no intencionales durante la cesárea (Ver Tabla 3).

\section{ANTECEDENTE DE CPPD Y CEFALEA}

El riesgo de un nuevo episodio de CPPD es 4,3 veces mayor en pacientes con antecedente previo de CPPD (OR 4,3; IC 95\% 1,99-9,31; p<0,01) 3,10. Lybecker $\mathrm{H}$ y cols, realizaron 117 punciones lumbares de los cuales cinco desarrollaron CPPD, dos de tres casos tenían antecedentes de CPPD y tres de 114 casos sin este antecedente, demostrando en su estudio que el antecedente de CPPD es un factor predictor significativo para la patología $(p=0,0018)^{24}$. Así mismo, la incidencia de CPPD es mayor en pacientes con antecedentes de cefalea, principalmente cefalea tensional ${ }^{25}$. Kuntz KM y cols, concluyeron que el grupo de pacientes con antecedentes de cefalea una semana previa a la punción dural tenía un incidencia de CPPD del $70 \%$. Por otro lado, un estudio prospectivo realizado en el 2013 por Van Oosterhout, W.P.J. y cols, encontraron que la migraña no incrementaba el riesgo de CPPD y que la punción dural no predisponía a ataques de migraña ${ }^{6}$. Adicionalmente, Webb CA y cols, realizaron un estudio de casos y controles concluyendo una mayor incidencia de cefalea crónica en el grupo de gestantes con puncion dural accidental (28\%) que en el grupo de control (5\%) (OR: 7; $p=0,0129)^{26}$.

\section{DISEÑO DE LA AGUJA ESPINAL}

Las agujas espinales que se utilizan para realizar las punciones durales pueden ser traumáticas (Quincke, Tuchy, Levi) o atraumáticas (Greene, Whitacre o punta de lápiz, Sprotte, Polymedic, Gertie Marx, Atraucan). La aguja espinal Quincke o punta de diamante, fue descrita en 1981 mientras que la aguja espinal atraumática Sprotte fue creada en Alemania en 1979 y es una modificación de la aguja espinal Whitacre ${ }^{27,28}$. Las agujas espinales atraumáticas tienen una punta en forma de cono circular cerrado con un perfil redondeado y una abertura lateral la cual es mayor que el diámetro interior ${ }^{27}$ separando las fibras de la duramadre, ocasionando menos perdida de LCR y por lo tanto menor incidencia de CPPD ${ }^{29-35}$; por el contrario, las agujas espinales traumáticas tienen una punta biselada que le permite realizar cortes en las diferentes capas de la duramadre. El costo de las agujas espinales atraumáticas era siete veces mayor que las agujas espinales traumáticas ${ }^{29,35}$, pero en los últimos 14 años su costo ha ido disminuyendo tanto inherente al procedimiento como al manejo de una posible complicación ${ }^{29,36,37}$.

En un estudio prospectivo aleatorio realizado en Israel en el 2004, fueron sometidos 58 pacientes a 85 punciones durales, comparando una aguja espinal traumática (Quincke calibre 22) versus una aguja espinal atraumática (Whitacre calibre 22), en donde se evidenció una incidencia del $36 \%$ en el grupo de pacientes que fueron sometidas a punciones durales con agujas traumáticas versus una incidencia del $3 \%$ con agujas espinales atraumáticas $(p=0,002)^{32}$. Adicionalmente, en Honduras fue realizado un estudio observacional en el mismo año en donde se estudiaron 50 pacientes embarazadas a quienes se les practicó cesárea bajo anestesia raquídea utilizando agujas espinales Whitacre en 25 pacientes y Quincke en el otro grupo de 25 pacientes. Se comparó la incidencia de CPPD entre ambos grupos a las 24,48 y 72 horas, encontrándose $8 \%$ de cefaleas después de 24 horas en el grupo en el que se utilizaron agujas espinales Whitacre y del $16 \%$ en el grupo con agujas Quincke. Se concluyó que el uso de la aguja espinal Whitacre es menos traumático para los tejidos de la duramadre, por su diseño no cortante en punta de lápiz ${ }^{38}$.

Un estudio prospectivo realizado por Hammond ER y cols entre el 2008 y el 2009 con 187 pacientes de consulta externa de neurología en la clínica Johns Hopkins indicó una prevalencia para CPPD del 32\% con las agujas espinales tipo Quincke y del 19\% con agujas tipo Sprotte. La probabilidad de CPPD aumentó cuatro veces cuando se utilizaba la aguja espinal Quincke calibre 22 en comparación con la aguja Sprotte del mismo calibre (OR: 3,98; IC 95\%:1,32-12,0; $\mathrm{p}=0,014)^{31}$. En otro estudio realizado por Müller $\mathrm{B}$ y cols, en el 1993 prospectivo doble ciego aleatorizado en 100 pacientes, se observó una incidencia del $31 \%$ en los pacientes sometidos a punción dural con agujas espinales traumáticas (Quincke calibre 20) en comparación con una incidencia del $5 \%$ con agujas espinales atraumáticas (Sprotte calibre 22) ${ }^{27}$. 


\section{CALIBRE DE LA AGUJA ESPINAL}

La frecuencia de CPPD reportada en el estudio por Turnbull DK y cols, fue del $70 \%$ si el tamaño de la aguja espinal era entre el calibre 16 y 19, 40\% si estaba entre el calibre 20 y 22 , y $12 \%$ si se encontraba entre el calibre 24 y $27^{38,39}$. López Correa T y cols, reportaron que la incidencia de CPPD era del $36 \%$ al utilizar agujas espinales Quincke calibre 22, mientras que al utilizar agujas espinales Quincke calibre 32, la diferencia era del 0,4\%39, así mismo Aamodt A y cols, encontraron que hay un riesgo significativo para CPPD con la aguja Quincke calibre 20 comparado con la aguja Withacre calibre $22^{40}$. Esto permite inferir que el calibre de la aguja espinal es directamente proporcional a la incidencia de CPPD 39 .

No obstante, hay estudios donde no hay diferencia estadísticamente significativa en el riesgo de padecer CPPD entre la utilización de la aguja espinal Quincke calibre 22 y la aguja Quincke calibre 2031; sin embargo, otros estudios con estos calibres de aguja reportan asociación significativa (50\% versus $26 \% ; \mathrm{P}=0,0002)^{21}$. Amorim JA y cols, refieren que no hay evidencia estadísticamente significativa entre la aguja espinal Quincke calibre 25 y la aguja espinal Quincke calibre $27^{3}$; de igual forma no existe evidencia estadísticamente significativa entre la utilización aguja Quincke calibre 29 y calibre $25^{41}$.

\section{ORIENTACIÓN DEL BISEL}

En un estudio epidemiológico prospectivo realizado por Lybecker H y cols en 1989, en el cual se practicaron 1021 punciones durales, se concluyó que la incidencia de CPPD es menor si la aguja es insertada con el bisel paralelo a las fibras de la duramadre ${ }^{24}$. Tarkkila y cols, reportaron una incidencia de CPPD con aguja Quincke con bisel perpendicular a la fibras de la duramadre del $17,9 \%$ y con aguja Quincke con bisel paralelo del 4,5\%42; por lo anterior la orientación del bisel perpendicular desencadena CPPD dos veces más que orientar el bisel en forma paralela (OR:2,16; IC: $1,07-4,35 ; p=0,03)^{3}$.

\section{REINSERCIÓN DEL ESTILETE}

En la realización de la punción dural se utiliza una guía, cánula exterior o mandril, con el fin de evitar doblar la fina aguja espinal en el ligamento supraespinoso ${ }^{27}$. Se ha evidenciado que la reinserción del estilete antes de la retirada de la aguja espinal puede facilitar el cierre dural reinsertando partículas del aracnoides en la duramadre y disminuyendo la perdida de LCR junto con la incidencia de CPPD29,31,43. En un estudio prospectivo aleatorizado en 1998 con 600 pacientes separados en dos grupos, en donde a uno de ellos se les reinserto el estilete se encontró una incidencia de CPPD en el $5 \%$ y $16,3 \%$ respectivamente ${ }^{44}$; sin embargo, un estudio en el 2013 prospectivo concluyó que no hay diferencia estadística en la reinserción del estilete ${ }^{45}$.

\section{NÚMERO DE INTENTOS POR PUNCIÓN Y EXPERIENCIA DE QUIEN REALIZA LA PUNCIÓN DURAL}

Hay estudios que reportan una asociación no significativa entre el número de intentos de punciones durales y la CPPD $6,24,31,32,34,46$. Sin embargo, Seeberger y cols realizaron un estudio prospectivo con 8034 punciones durales encontrando asociación estadística, estos autores atribuyen la falta de consistencia de los resultados en estudios anteriores debido a un bajo poder estadístico ${ }^{47}$. Pese a la disminución del riesgo de CPPD al utilizar agujas espinales de menor calibre, estas requieren de más destreza; por lo tanto es importante la experiencia de quien realiza el procedimiento, debido al mayor número de fallas por la dificultad técnica que presenta $^{27,38}$. No se ha observado diferencia en el desarrollo de CPPD luego de que el procedimiento sea realizado por una enfermera, un médico, un residente o un estudiante de medicina entrenado en la técnica ${ }^{6,7}$, al igual que no hay diferencia estadística entre la experiencia de los anestesiólogos ${ }^{45}$.

\section{OTROS FACTORES}

Dodge HS y cols, en su estudio en el 2013 concluyeron que la diferencia de las tasas de CPPD entre los caucásicos y los afroamericanos (28\% versus $15,6 \%$ ) no fueron significativas $(p=0,18)^{16}$, estas mismas diferencias en la raza también fueron encontradas por Almeida SM y cols ${ }^{8}$, por lo cual la raza no ha demostrado ser factor de riesgo para CPPD ${ }^{15}$. Por otro lado, no hay evidencia científica de que el embarazo por sí solo sea un factor de riesgo para CPPD; sin embargo, la alta incidencia en este grupo poblacional podría estar relacionada con otros factores como la edad, sexo y la punciones durales accidentales ${ }^{39}$. De igual forma, el estudio retrospectivo realizado por Dodge HS y cols, en el 2013 en 153 pacientes encontró que los fumadores podrían asociarse a una incidencia reducida de CPPD comparada con los no fumadores $(13,7 \% \text { versus. } 34,1 \% ; p=0,009)^{16}$; aunque en otros 
estudios esta incidencia no es significativa ${ }^{42}$. En este mismo estudio se encontró que el trastorno de estrés post traumático podría ser un factor protector en el desarrollo de CPPD ${ }^{16}$.

Del mismo modo, no hay diferencia estadísticamente significativa de la prevalencia de CPPD entre reposo en decúbito supino y deambulación precoz posterior a la punción dural ${ }^{46,48,49}$. Así mismo, un estudio prospectivo realizado en una muestra pequeña de tan solo 59 pacientes encontró que la cantidad de LCR y la hidratación endovenosa no influyen en la aparición de CPPD ${ }^{49}$. El abordaje de la punción dural puede ser medial o páramedial, no existen estudios concluyentes que evidencien que una técnica sea mejor que la otra ${ }^{39}$, adicionalmente no hay asociación estadísticamente significativa entre la CPPD y el tiempo en el que permanece sentado el paciente después de la punción dural y antes de procedimiento quirúrgico ${ }^{50}$ (Ver Tabla 3).

Tabla 3. Factores de riesgo y protectores para CPPDa

\begin{tabular}{|c|c|c|}
\hline Factor de riesgo & OR (IC) & $\mathbf{p}$ \\
\hline Edad $(31-50 \text { años })^{3}$ & $2,21(1,12-4,36)^{b}$ & 0,02 \\
\hline Sexo femenino ${ }^{3}$ & $2,25(1,07-4,73)^{b}$ & 0,03 \\
\hline Antecedente personal de CPPD ${ }^{3}$ & $4,3(1,99-9,31)^{b}$ & $<0,01$ \\
\hline Aguja traumática (Quincke vs Sprotte) 30 & $3,98(1,32-12,0)^{b}$ & 0,014 \\
\hline Orientación del bisel perpendicular ${ }^{3}$ & $2,16(1,07-4,35)^{b}$ & 0,03 \\
\hline ¿IMCc bajo $\left(\leq 25 \mathrm{Kg} / \mathrm{m}^{2} \text { vs }>25 \mathrm{Kg} / \mathrm{m}^{2}\right)^{7} ?$ & $3,3(1,5-7,0)$ & 0,001 \\
\hline ¿Antecedente personal de cefalea? & - & - \\
\hline ¿Mayor calibre de la aguja? & - & - \\
\hline ¿No reinserción del estilete? & - & - \\
\hline ¿Mayor número de intentos por punción? & - & - \\
\hline Factores protectores & OR (IC) & $\mathbf{p}$ \\
\hline ¿Tabaquismo? & - & - \\
\hline ¿Trastorno de estrés post traumático? & - & - \\
\hline
\end{tabular}

${ }^{a}$ cefalea post punción dural, bodds ratio ajustado, cindice de masa corporal Fuente: Datos tomados de Amorim JA y $\mathrm{col}^{3}$, Almeida SM y col${ }^{7}$ y Hammond ER y col $\mathrm{Ha}^{30}$.

\section{CONCLUSIONES}

Los factores de riesgo para desarrollar cefalea pospunción dural pueden ser clasificados en dos grupos: aquellos factores modificables, relacionados con el insumo y el procedimiento, como la aguja traumática, orientación del bisel perpendicular, mayor calibre de la aguja, no reinserción del estilete y mayor número de intentos por punción, y aquellos factores no modificables tales como edad entre los 31 y 50 años, sexo femenino, antecedente de cefalea pospunción dural y bajo peso $(\mathrm{IMC}<25 \mathrm{Kg} /$ $\mathrm{m}^{2}$ ). La edad entre 31 a 50 años, sexo femenino, antecedente personal de cefalea pospunción dural, aguja traumática y orientación del bisel perpendicular son factores de riesgos con significancia estadística, mientras que para los factores como bajo peso, antecedente personal de cefalea, mayor calibre el aguja, no reinserción del estilete y mayor número de intentos por punción, los resultados de los estudios no son consistentes.

Estrategias para prevenir la cefalea pospunción dural serían la protocolización de la punción dural en la que se puedan intervenir factores de riesgo relacionados con el insumo y el procedimiento. Adicionalmente, se podría realizar un score que permita predecir que pacientes tienen una alta probabilidad de desarrollar cefalea pospunción dural e iniciar tratamientos profilácticos. Se requieren más estudios de tipo prospectivo que permitan aclarar la asociación de estos factores de riesgo con la cefalea pospunción dural. Hasta el momento no hay investigaciones que utilicen los criterios diagnósticos de la clasificación internacional de desórdenes de cefalea tercera edición (versión beta). 


\section{Referencias Bibliográficas}

1. Gaiser RR. Postdural puncture headache: a headache for the patient and a headache for the anesthesiologist. Current Opinion in Anaesthesiology. 2013;26(3):296-303.

2. Alstadhaug KB, Odeh F, Baloch FK, Berg DH, Salvesen R. Post-lumbar puncture headache. Tidsskr Nor Laegeforen. 2012;132(7):818-21.

3. Amorim JA, Gomes de Barros MV, Valença MM. Post-dural (postlumbar) puncture headache: risk factors and clinical features. Cephalalgia. 2012;32(12):916-23.

4. Bezov D, Ashina S, Lipton R. Post-Dural Puncture Headache: Part II - Prevention, Management, and Prognosis. Headache. 2010;50(9):1482-1498.

5. Kuczkowski KM. Postdural puncture headache after lumbar puncture: Do the gauge and the design of a spinal needle matter? Am J Emerg Med. 2006;24(6):757.

6. van Oosterhout WP, van der Plas AA, van Zwet EW, Zielman R, Ferrari MD, Terwindt GM. Postdural puncture headache in migraineurs and nonheadache subjects: a prospective study. Neurology. 2013;80(10):941-8.

7. Kuntz KM, Kokmen E, Stevens JC, Miller P, Offord KP, Ho MM. Post-lumbar puncture headaches: experience in 501 consecutive procedures. Neurology. 1992;42(10):1884-7.

8. de Almeida SM, Shumaker SD, LeBlanc SK, Delaney P, MarquieBeck J, Ueland S, et al. Incidence of post-dural puncture headache in research volunteers. Headache. 2011;51(10):1503-10.

9. Harrington BE. Postdural puncture headache and the development of the epidural blood patch. Reg Anesth Pain Med. 2004;29(2):136-63.

10. Amorim JA, Valença MM. Postdural puncture headache is a risk factor for new postdural puncture headache. Cephalalgia. 2008;28(1):5-8.

11. Singh S, Chaudry SY, Phelps AL, Vallejo MC. A 5-year audit of accidental dural punctures, postdural puncture headaches, and failed regional anesthetics at a tertiary-care medical center. TheScientificWorldJournal. 2009; 9:715-722.

12. International Headache Society. The International Classification of Headache Disorders, 3rd edition (beta version). Cephalalgia. 2013;33(9):629-808.

13. Headache Classification Subcommittee of the International Headache Society. The International Classification of Headache Disorders: 2nd edition. Cephalalgia. 2004; 24 (suppl 1):79.

14. Calderon R, Copenhaver D. Postdural puncture headache. Journal of pain \& palliative care pharmacotherapy. 2013;27(4):406-407.

15. Bezov D, Lipton RB, Ashina S. Post-Dural Puncture Headache: Part I Diagnosis, Epidemiology, Etiology, and Pathophysiology. Headache. 2010 ;50(7):1144-1152

16. Dodge HS, Ekhator NN, Jefferson-Wilson L, Fischer M, Jansen I, Horn PS, et al. Cigarette smokers have reduced risk for post-dural puncture headache. Pain physician. 2013;16:E25-E30

17. Zetterberg $\mathrm{H}$, Tullhög $\mathrm{K}$, Hansson $\mathrm{O}$, Minthon $\mathrm{L}$, Londos $\mathrm{E}$, Blennow K. Low incidence of post-lumbar puncture headache in 1,089 consecutive memory clinic patients. Eur Neurol. 2010;63(6):326-330.

18. Janssens E, Aerssens P, Alliët P, Gillis P, Raes M. Post-dural puncture headaches in children. A literature review. Eur J Pediatr. 2003;162(3):117-21.

19. Ramamoorthy C, Geiduschek JM, Bratton SL, Miser AW, Miser JS. Postdural puncture headache in pediatric oncology patients. Clin Pediatr. 1998;37(4):247-51.

20. Wu CL, Rowlingson AJ, Cohen SR, Michaels RK, Courpas GE, Joe EM, et al. Gender and post-dural puncture headache. Anesthesiology. 2006;105(3):613-18.

21. Vilming ST, Kloster R, Sandvik L. The importance of sex, age, needle size, height and body mass index in post-lumbar puncture headache. Cephalalgia. 2001;21(7):738-43

22. Vricella LK, Louis JM, Mercer BM, Bolden N. Anesthesia complications during scheduled cesarean delivery for morbidly obese women. Obstet Gynecol. 2010;203(3):276.e1-5.

23. Faure E, Moreno R, Thisted R. Incidence of postdural puncture headache in morbidly obese parturients. Reg Anesth. 1994;19(5):361-3.

24. Lybecker H, Møller JT, May O, Nielsen HK. Incidence and prediction of postdural puncture headache. A prospective study of 1021 spinal anesthesias. Anesth Analg. 1990;70(4):389-94.

25. Nowaczewska M, Ksikiewicz B. Cerebral blood flow characteristics in patients with post-lumbar puncture headache. J Neurol. 2012;259(4):665-9.

26. Webb CA, Weyker PD, Zhang L, Stanley S, Coyle DT, Tang T, et al. Unintentional dural puncture with a Tuohy needle increases risk of chronic headache. Anesth Analg. 2012;115(1):124-32.

27. Müller B, Adelt K, Reichmann H, Toyka K. Atraumatic needle reduces the incidence of post-lumbar puncture syndrome. J Neurol. 1994; 241(6):376-80.

28. Birnbach DJ, Kuroda MM, Sternman D, Thys DM. Use of atraumatic spinal needles among neurologists in the United States. Headache. 2001;41(4):385-90.

29. Lavi R, Rowe JM, Avivi I. Lumbar Puncture: It Is Time to Change the Needle. Eur Neurol. 2010;64(2):108-13.

30. Lowery S, Oliver A. Incidence of postdural puncture headache and backache following diagnostic/therapeutic lumbar puncture using a $22 \mathrm{G}$ cutting spinal needle, and after introduction of a $25 \mathrm{G}$ pencil point spinal needle. Paediatr Anaesth. 2008;18(3):230-4.

31. Hammond ER, Wang Z, Bhulani N, McArthur JC, Levy M. Needle type and the risk of post-lumbar puncture headache in the outpatient neurology clinic. J Neurol Sci. 2011;306(1-2):24-8.

32. Lavi R, Yarnitsky D, Rowe JM, Weissman A, Segal D, Avivi I. Standard vs atraumatic Whitacre needle for diagnostic lumbar puncture: a randomized trial. Neurology. 2006;67(8):1492-4.

33. Strupp M, Schueler O, Straube A, Von Stuckrad-Barre S, Brandt T. "Atraumatic" Sprotte needle reduces the incidence of postlumbar puncture headaches. Neurology. 2001;57(12):2310-2.

34. Apiliogullari S, Duman A, Gok F, Akillioglu I. Spinal needle design and size affect the incidence of postdural puncture headache in children. Paediatr Anaesth. 2010;20(2):177-82.

35. Thomas SR, Jamieson DRS, Muir KW. Randomised controlled trial of atraumatic versus standard needles for diagnostic lumbar puncture. Br Med J. 2000;321(7267):986-90.

36. Davis A, Dobson R, Kaninia S, Espasandin M, Berg A, Giovannoni $\mathrm{G}$, et al. Change practice now! Using atraumatic needles to prevent post lumbar puncture headache. EUR J NEUROL. 2014;21(2):305-11.

37. Tung CE, So YT, Lansberg MG. Cost comparison between the atraumatic and cutting lumbar puncture needles. Neurology. 2012;78(2):109-13

38. Samayoa F, Ramos N, Sánchez A. Cefalea post punción dural al utilizar agujas de Quincke vrs. agujas de Whitacre en pacientes obstétricas. Rev. Colomb. Anestesiol. 2004;32:253-60.

39. López Correa T, Garzón Sánchez JC, Sánchez Montero FJ, Muriel Villoria C. Postdural puncture headache in obstetrics. REDAR . 2011;58(9):563-73.

40. Aamodt A, Vedeler C. Complications after LP related to needle type: pencil-point versus Quincke. Acta Neurol Scand. 2001;103(6):396-98.

41. Schmittner MD, Terboven T, Dluzak M, Janke A, Limmer ME, Weiss C, et al. High incidence of post-dural puncture headache in patients with spinal saddle block induced with Quincke needles for anorectal surgery: a randomised clinical trial. Int J Colorectal Dis. 2010;25(6):775-81.

42. Tarkkila PJ, Heine H, Tervo RR. Comparison of Sprotte and Quincke needles with respect to post dural puncture headache and backache. Reg Anesth 1992;17(5):283-87.

43. Strupp M, Brandt T. Should One Reinsert the Stylet during Lumbar Puncture?. N Engl J Med. 1997;336(16):1190.

44. Strupp M, Brandt T, Müller A. Incidence of post-lumbar puncture syndrome reduced by reinserting the stylet: a randomized prospective study of 600 patients. J Neurol. 1998;245(9):589-92.

45. Sinikoglu NS, Yeter H, Gumus F, Belli E, Alagol A, Turan N. Reinsertion of the stylet does not affect incidence of post dural Puncture Headaches (PDPH) after spinal anesthesia. Braz. j. anesthesiol. 2013;63(2):188-92.

46. Kim SR, Chae HS, Yoon MJ, Han JH, Cho KJ, Chung SJ. No effect of recumbency duration on the occurrence of post-lumbar puncture headache with a $22 \mathrm{G}$ cutting needle. BMC Neurology. 2012;12:1.

47. Seeberger MD, Kaufmann M, Staender S, Schneider M, Scheidegger D. Repeated dural punctures increase the incidence of postdural puncture headache. Anesth Analg. 1996;82(2):302-5.

48. Tejavanija S, Sithinamsuwan P, Sithinamsuwan N, Nidhinandana S, Suwantamee J. Comparison of prevalence 
of post-dural puncture headache between six hour- supine recumbence and early ambulation after lumbar puncture in thai patients: A randomized controlled study. J. Med. Assoc. Thai. 2006;89(6):814-20.

49. Bauset-Navarro J, Sánchez-Ortuño I.M, Gómez-Cárdenas C, Sanz-Monllor A, Cinesi-Gómez C, Piñera-Salmerón P. Iatrogenic after spinal puncture technique. Prevalence study of headache and associated factors. Rev. Neurol. 2014;58(5):193-8.

50. Schmittner MD, Urban N, Janke A, Weiss C, Bussen DG, Burmeister MA, et al. Influence of the pre-operative time in upright sitting position and the needle type on the incidence of post-dural puncture headache (PDPH) in patients receiving a spinal saddle block for anorectal surgery. Int. J. Colorectal. Dis. 2011;26(1):97-102. 